Pacific Journal of Mathematic 


\title{
NORMAL SUBSPACES OF THE DENSITY TOPOLOGY
}

\author{
FrankLin D. TALl
}

The density topology on the real line is a strengthening of the usual Euclidean topology which is intimately connected with the measure-theoretic structure. The space itself is not normal; we are interested in characterizing its normal subspaces. This leads us to the consideration of various set-theoretic axioms, and yields a consistent example of a homogeneous normal non-collectionwise Hausdorff space and indeed a general method for producing normal non-collectionwise Hausdorff spaces. (A space is collectionwise Hausdorf if for each closed discrete subset $Y$ there exist pairwise disjoint open sets, one about each element of $Y$.)

A familiarity with [24] - a comprehensive collection of facts about the density topology - will be useful although not entirely essential in perusing this paper. Recall that the open sets of the density topology $\mathfrak{F}$ are those measurable sets $U$ such that for each $x \in U$,

$$
\lim _{h \rightarrow 0} \frac{m(U \cap[x-h, x+h])}{2 h}=1 .
$$

We state for future reference the crucial properties of this topology.

THEOREM 1. Let $X$ be the real line with the density topology.

(1) $Y \subseteq X$ is a nullset (i.e., has measure 0) iff it is nowhere dense iff it is closed discrete,

(2) $X$ has a basis of cardinality $2^{\boldsymbol{N}_{0}}$,

(3) Borel subsets of $X$ are measurable and are $G_{\delta}$ 's,

(4) $X$ is completely regular,

(5) every subspace of $X$ is the union of a closed discrete subspace and a subspace satisfying the countable chain condition (disjoint collections of open sets are countable).

Definition 2. A set $\boldsymbol{S}$ of reals is a Sierpinski set if it has countable intersection with every nullset. $S$ is a generalized Sierpinski set if its intersection with every nullset has cardinality less than continuum.

It is well-known-and we will prove below-that Martin's Axiom [15] entails the existence of generalized Sierpiński sets of cardinality continuum, and (hence) the continuum hypothesis yields Sierpiński sets of that cardinality.

In [24] we proved the following results. 
THEOREM 3. If $Y \subseteq X$ (the real line with the density topology) is a Sierpinski set, $Y$ is hereditarily Lindelof and hence (since $X$ is regular) normal. If $Y$ is normal, then $Y$ is the union of a generalized Sierpinski set and a nullset. If $2^{\boldsymbol{N}_{0}}<2^{\boldsymbol{*}_{1}}$ and $Y$ is normal, $Y$ is the union of a Sierpinski set and a nullset.

Upon closer analysis of the proof in [24] one obtains a slightly strengthened version of the consequences of normality:

Lemma. If $Y \subseteq X$ is normal then $Y$ is the union of a generalized Sierpinski set $S$ and $a$ nullset $T$ such that $\bar{S} \cap T=\varnothing$.

Proof. $Y=(Y \cap \overline{\text { int } \bar{Y}}) \cup(Y-\overline{\text { int } \bar{Y}})$. The closure of the first half is disjoint from the second half. The second half is a nullset. Since $Y \cap$ int $\bar{Y}$ is dense in int $\bar{Y}$ and in $Y \cap \overline{\text { int }} \bar{Y}$, all three have isomorphic regular open algebras. But $\mid R O$ (int $\bar{Y})|\leqq| R O(X) \mid \leqq 2^{\varkappa_{0}}$, so arguing as in [24] we get that if $Y$ is normal then $Y \cap$ int $\bar{Y}$ is generalized Sierpinski.

Examination of this proof shows that the union of a non-trivial (generalized) Sierpiński set and a nullset need not be normal. For if there is a generalized Sierpiński set of power continuum, by Theorem $1(5)$ there is one-call it $S$-which satisfies the countable chain condition. $\bar{S}$ is measurable and so includes a nullset $T$ of power continuum. $S \cup T$ is not normal since $|R O(S \cup T)| \leqq 2^{\kappa_{0}}$. We shall see later that generalized Sierpiński sets themselves need not be normal. But under reasonable set-theoretic hypotheses we do get a characterization:

Theorem 4. Assume $2^{\aleph_{0}}<2^{\aleph_{1}}$ (respectively, Martin's Axiom). Then $Y \subseteq X$ is normal if and only if $Y$ is the union of a Sierpiniski (respectively, generalized Sierpinski) set $S$ and a nullset $T$ such that $\bar{S} \cap T=\varnothing$.

Both forward directions have been proved. In proving the backward directions, $T$ may be ignored since it is normal and clopen in $S \cup T$. Then Theorem 3 disposes of the $2^{\boldsymbol{N}_{0}}<2^{\boldsymbol{k}_{1}}$ case. To finish the other case, we detour to study generalized Lusin spaces.

DEFINITION 5. A generalized Lusin space is a space in which every nowhere dense subset has cardinality $<2^{\boldsymbol{N}_{0}}$.

A generalized Sierpiński set as a subspace of the density topology is thus a generalized Lusin space. Also, assuming Martin's Axiom or the continuum hypothesis, it is well known that there exist generalized Lusin subspaces of the real line having cardinality continuum. We shall improve this result later. 
Definition 6. A space $\langle X, \mathfrak{F}\rangle$ is cometrizable if there exists a weaker separable metrizable topology $\mu$ on $X$ such that " $₹$ is regular with respect to $\mathcal{M}$ ", i.e. whenever $x \in U \in \mathfrak{\mho}$, there is a $V \in \mathfrak{F}$ such that $x \in V \subseteq \operatorname{cl}_{\mathcal{M}}(V) \subseteq U$, where $\operatorname{cl}_{\mathcal{M}}(V)$ is the closure of $V$ in $\mathcal{M}$.

For example,

LEMMA. The density topology is cometrizable.

The proof that the density topology is regular in [16] actually establishes the stronger regularity condition mentioned above.

The importance of cometrizability in the study of normality was highlighted by the following important result.

TheOREM 7 [1]. Martin's Axiom implies every cometrizable space of cardinality $<2^{\aleph_{0}}$ is normal.

In fact their proof establishes

THEOREM 8. Martin's Axiom implies that if $F, G$ are disjoint closed sets of cardinality $<2^{\aleph_{0}}$ in a cometrizable space, there exist disjoint open sets about them.

Since in order to separate two disjoint closed sets it suffices to separate their boundaries, it is evident that generalized Lusin subspaces of cometrizable spaces are normal, assuming Martin's Axiom. (In fact such subspaces are hereditarily normal, since the properties of being generalized Lusin or cometrizable are both easily seen to be hereditary.) Thus the proof of Theorem 4 is completed. We remain with generalized Lusin spaces long enough to establish sufficient conditions under which they exist. These will yield the already alluded to "well-known fact" that generalized Sierpiński sets exist, assuming Martin's Axiom, and produce other interesting-in particular noncollectionwise Hausdorff-examples as well [25], [4]. We are using cometrizability heavily, but a surprisingly wide class of spaces satisfies this condition [18].

Definition 9. A space is Baire if no nonempty open set is the union of countably many nowhere dense sets. A space is strong Baire if no nonempty open set is the union of $<2^{x_{0}}$ nowhere dense sets. Martin's Axiom works for a (Baire) space if, under the assumption of Martin's Axiom, the space is strong Baire. The $\pi$-weight of a space $X, \pi(x)$, is the least cardinal of a collection (called a $\pi$-base) of non-empty open sets such that every non-empty open set includes one.

In [8] it is shown that the continuum hypothesis ensures the existence of dense generalized Lusin spaces of power continuum in every 
countable chain condition Baire space without isolated points having $\pi$-weight $\leqq 2^{N_{0}}$. The same proof yields

Theorem 10. Assume Martin's Axiom. Then every countable chain condition space $X$ without isolated points having $\pi$-weight $\leqq 2^{\aleph_{0}}$ for which Martin's Axiom works has a dense generalized Lusin subspace Y of cardinality $2^{\mathrm{N}_{0}}$.

Thus Martin's Axiom yields generalized Sierpiński sets of cardinality continuum.

It should be noted that the restriction on isolated points is needed only to insure that $Y$ has cardinality continuum. It is useful to have $Y$ dense, since this ensures that it satisfies the countable chain condition. We are of course interested in the case when $X$ is cometrizable. In this case we may drop the $\pi$-weight restriction:

LEMMA. If $X$ is cometrizable, $\pi(X) \leqq 2^{\boldsymbol{\aleph}_{0}}$.

Proof. The (non-empty) interiors of sets closed in the separable metrizable topology form a $\pi$-base. Note that a separable metric space has no more than continuum closed sets.

At the cost of omitting "dense" it can be assumed that the generalized Lusin space includes no open sets of cardinality $<2^{N_{0}}$ and hence is strong Baire. More precisely:

THEOREM 11. Suppose $Y$ is a countable chain condition generalized Lusin space of power continuum. Then there is an open subspace $Y^{\prime}$ of $Y$ (hence $Y^{\prime}$ is countable chain condition and generalized Lusin) which has cardinality continuum and includes no open sets of cardinality less than continuum. If $2^{\aleph_{0}}$ is a regular cardinal (as e.g. under Martin's Axiom [15]), $Y^{\prime}$ is strong Baire.

Proof. Suppose on the contrary there is a $\pi$-base of open sets of cardinality $<2^{\mathbf{N}_{0}}$. Take a maximal disjoint collection of such open sets. Its union and remainder decompose $Y$ into countably many sets of cardinality $<2^{\boldsymbol{\kappa}_{0}}$. But $2^{\boldsymbol{\kappa}_{0}}$ does not have countable cofinality. Similarly, if $2^{\boldsymbol{x}_{0}}$ is a regular cardinal, an open set of cardinality continuum cannot be the union of a small number of small nowhere dense sets.

In previous preprints I have mistakenly claimed all non-trivial generalized Lusin spaces are strong Baire if $2^{\kappa_{0}}$ is regular. This is true for the density topology, assuming Martin's Axiom, because nowhere dense sets are discrete. In general, however, this need not be true. I am grateful to the referee for pointing out that one could consider e.g. the disjoint union of the positive rationals with a generalized Lusin 
subspace of the negative reals. Fortunately, all the uses to which I put this claim go through via Theorem 11 .

Given a dense generalized Lusin subspace $Y$ of a space $X$, the adjunction of any set $Z$ of fewer than $2^{\boldsymbol{k}_{0}}$ points of $X$ to $Y$ produces another dense generalized Lusin subspace $Y^{\prime}=Y \cup Z$. If $Y$ is countable chain condition and $Z$ is uncountable closed discrete, $Y^{\prime}$ is not collectionwise Hausdorff. We thus have

Theorem 12. Assume Martin's Axiom plus $2^{\mathrm{N}_{0}}>\boldsymbol{N}_{1}$. Suppose $X$ satisfies the following conditions:

(1) countable chain condition,

(2) no isolated points,

(3) Martin's Axiom works,

(4) uncountable closed discrete subspace,

(5) cometrizable.

Then $X$ has a dense hereditarily normal non-collectionwise Hausdorff subspace.

The large number of conditions in Theorem 11 should raise the question of whether any $X$ satisfies them. In fact at least two interesting spaces do. The first space is the Pixley-Roy topology on compact nowhere dense sets of reals [8]. Verification of the details appears in [25]; here we wish only to mention that the resulting generalized Lusin space is countable chain condition, non-separable, first countable, and, applying Theorem 11 if necessary, (strong) Baire. No such normal non-collectionwise Hausdorff space was previously known.

The second space satisfying the conditions of Theorem 12 is of course the density topology. In this case it is not necessary to adjoin a set $Z$ to the generalized Lusin set since Martin's Axiom implies subsets of the density topology of power $<2^{N_{0}}$ are closed discrete.

It is not quite so easy to construct a generalized Sierpiński set which is first category on the real line and is an additive subgroup of the reals, but this too can be done with Martin's Axiom [26]. The resulting subspace of the density topology has a remarkable collection of properties. The first eight hold for arbitrary generalized Sierpiński sets, assuming Martin's Axiom.

TheOREM 13. Assume Martin's Axiom. Then there is a space $S$ such that

(1) $|S|=2^{\boldsymbol{x}_{0}}$

(2) every subspace of $S$ of cardinality $<2^{N_{0}}$ is closed discrete,

(3) every discrete subspace of $S$ is closed,

(4) every dense subset of $X$ has cardinality $2^{\boldsymbol{N}_{0}}$,

(5) the union of any collection of open sets is equal to the union of a subcollection of power $<2^{\kappa_{0}}$, 
(6) $S$ is hereditarily Baire and hereditarily subparacompact,

(7) $S$ is perfectly normal,

(8) if $2^{n_{0}}>N_{1}, S$ is not collectionwise Hausdorf,

(9) $S$ satisfies the countable chain condition,

(10) $S$ is 0-dimensional,

(11) if $2^{\varkappa_{0}}>\aleph_{1}, S$ has caliber $\aleph_{1}$,

(12) $S$ is homogeneous,

(13) $S^{2}$ is first category in $S^{2}$.

Proof. (3) and (6) hold for $S$ since they do for $X$ [24]. (1) is by construction. (2) holds since Martin's Axiom implies sets of power $<2^{\boldsymbol{x}_{0}}$ are nullsets. (4) follows from (2). (7) follows from Theorem 4 since closed subsets of $X$ are $G_{\delta}$ 's. To see (5), recall any set $T$ is the union of a set $T_{1}$ satisfying the countable chain condition and a nullset $T_{2}$. If $T$ is included in a union of open sets, countably many will cover $T_{1}$ except for a nowhere dense set $T_{3}$. Since $T$ is included in a generalized Sierpiński set, fewer than continuum many will cover $T_{1}$ and $T_{3}$. To prove (8), assume $2^{N_{0}}>N_{1}$. The countable chain condition piece of $S$ includes a (closed discrete) subset of power $\aleph_{1}$ which cannot be separated by disjoint open sets. (9) clearly doesn't hold for arbitrary generalized Sierpiński sets (throw in an uncountable nullset), but follows e.g. from (12). Namely the countable chain condition part of $S$ must be dense in $S$, else $S$ would have isolated points, whence $S$ would be discrete. The proof in [14] that Lusin spaces are 0-dimensional actually establishes that any countable chain condition completely regular space in which nowhere dense sets have power $<2^{\kappa_{0}}$ is 0 -dimensional. Thus (10). In [23] it is shown that in a countable chain condition space in which the union of $\aleph_{1}$ nowhere dense sets is nowhere dense, point-countable collections of open sets are countable. This establishes (11). (12) and (13) are proved in [26].

The interest in (4) and (5) is that, even for the class of perfectly normal spaces, the conjecture that Martin's Axiom plus the negation of the continuum hypothesis implies that "hereditary density" = "hereditary covering number" if one is less than continuum, fails.

Hajnal and Juhász [12] ask for an example of a regular space in which discrete subspaces are closed. $X$ is one. They construct a rather complicated consistent example of a perfectly normal space with this property, while ours follows easily from Martin's Axiom plus $2^{\kappa_{0}}>$ $\boldsymbol{N}_{1}$. (We have recently learned that van Douwen [6] has a countable such space.)

$S$ is quite different from other normal non-collectionwise Hausdorff spaces [2], [3], [5], [9], [11], [17], [19], [22], etc. by virtue of its homogeneity. Any subspace of power continuum is normal but not collectionwise Hausdorff. This contrasts sharply with other normal 
non-collectionwise Hausdorff spaces which are the union of two or $\aleph_{0}$ "nice" spaces, where nice means e.g. discrete or metrizable or paracompact. (For the reader unfamiliar with these spaces, it should be mentioned that they are of interest in connection with the normal Moore space conjecture.)

Getting back to our original question we still do not have an "absolute" characterization of the normal subspaces of $X$. However I propose the following

ConJecture. $Y \subseteq X$ is normal if and only if $Y=S \cup T$, where $S$ is generalized Sierpiński, $T$ is a nullset such that $\bar{S} \cap T=\varnothing$, and for every nullset $Z \subseteq S$, every subset of $Z$ is the intersection of $Z$ with a Euclidean $F_{\text {o } \delta}$.

I can prove the forward direction of the Conjecture. The point is that since $Z$ is closed discrete, any subset of $Z$ is closed, hence can be separated from its complement in $Z$ by open subsets of $X$ intersected with $S$. These open sets can be taken to be regular open, but by [20] such sets are Euclidean $F_{\sigma \delta}$. As for the other direction, to prove $Y$ normal it clearly suffices to prove $S$ normal. To separate two disjoint closed sets, it suffices to separate their boundaries, so indeed normality of $S$ reduces to the problem of separating a subset of a nullset (the union of the two boundaries) from the rest of the nullset. Note that under the assumption that every set of power $<2^{\varkappa_{0}}$ has measure 0 , we can replace the requirement that $Z$ be null by the stipulation that the cardinality of $Z$ be less than continuum.

One reason the Conjecture is attractive is that the normality of another strengthening of the Euclidean topology (the bubble space on a set of reals) is equivalent to the existence of an uncountable set of reals $Z$ such that every subset of $Z$ is the intersection of $Z$ with a Euclidean $F_{\sigma}$. See Chapter III of [21] for discussion. Assuming Martin's Axiom, every set $Z$ of reals of power less than continuum has this property. There are a number of interesting questions about $F_{\sigma}$ 's and $F_{\sigma \delta}$ 's that present set-theoretic methods do not provide an avenue of attack on-e.g. if a set of reals has in its relative topology every subset $F_{\sigma \delta}$, must in fact every subset be $F_{\sigma}$ ?

The following remarks are addressed to the reader familiar with models of set theory and arise from conversations with $\mathrm{K}$. Kunen to whom we are grateful.

Consider a model in which Martin's Axiom holds while $2^{\mathbf{x}_{0}}$ is inaccessible. Then a generalized Sierpinski set of power continuum is an example of "sup $\neq$ max" [13] for various cardinal functions.

Refer to Theorem 3. In a model of Martin's Axiom plus $2^{\boldsymbol{N}_{0}}>\boldsymbol{N}_{1}$, there are generalized Sierpiński sets but no Sierpiński sets; therefore normal does not imply Sierpiński. Adjoin $\aleph_{2}$ random reals to a model of 
$2^{\boldsymbol{N}_{0}}=\boldsymbol{\aleph}_{1}$ and $2^{\boldsymbol{\aleph}_{\mathrm{t}}}=\boldsymbol{\aleph}_{3}$. In the resulting model the union of $<2^{\boldsymbol{\aleph}_{\mathrm{o}}}$ nullsets does not cover the real line, and hence there is a generalized Sierpiński set $S$ of power continuum. $S$ is not normal however, since $2^{\boldsymbol{N}_{0}}<2^{\boldsymbol{\kappa}_{1}}$.

Since this note is about the density topology, we take this opportunity to answer a question of van Douwen although it is unrelated to normality.

DefinItION [6]. A neighborhood assignment for a space $\langle X, \mathfrak{F}\rangle$ is a function $\Phi: X \rightarrow \Im$ such that $x \in \Phi(x)$. A space $X$ is a $D$-space if for every neighborhood assignment $\Phi$ for $X$ there is a closed discrete $D \subseteq X$ such that $\cup \Phi[D]=X$, where $\Phi[D]=\{\Phi(x): x \in D\}$.

\section{THEOREM 14. The density topology is (hereditarily) a D-space.}

Proof. The result follows easily from (1) and (5) of Theorem 1. Namely given a neighborhood assignment $\Phi$ for $Y \subseteq X$, find points $\left\{x_{n}\right\}_{n<\omega}$ and disjoint open sets $\left\{U_{n}\right\}_{n<\omega}$ such that $x_{n} \in U_{n} \subseteq \Phi\left(x_{n}\right)$ and $\cup\left\{U_{n}: n<\omega\right\}$ is dense in $Y$. Then $\left\{x_{n}: n<\omega\right\} \cup\left(Y-\cup\left(U_{n}: n<\omega\right\}\right)$ is the required $D$.

In response, van Douwen has answered our question in [24] by showing that the density topology is not pseudonormal and has kindly given us permission to include his proof. This result has also be proved by L. Zajiček and J. Lukeš.

\section{THEOREM 15. The density topology is not pseudonormal.}

Proof. (van Douwen). In [10] it is shown that if $A, B \subseteq \mathbf{R}$ are disjoint and dense in the Euclidean topology, then there is no $\mathfrak{乛}$ continuous real-valued function which is 1 on $A$ and 0 on $B$. In particular, let $\boldsymbol{A}=\mathbf{Q}$ and $B=\{q+\sqrt{2}: q \in \mathbf{Q}\}$. Using pseudonormality one could obtain a $\mathfrak{\mho}$-discrete family of $\mathfrak{\Im}$-open sets $\left\{U_{a}: a \in A\right\}$ such that $a \in U_{a}$ and $U_{a} \cap B=\varnothing$ for all $a \in A$. By the complete regularity of $\mathfrak{F}$ there would then exist $\mathfrak{F}$-continuous $f_{a}: \mathbf{R} \rightarrow \mathbf{R}$ such that $f_{a}(a)=1$ and $f_{a}\left(\mathbf{R}-U_{a}\right)=0$. Then $\Sigma\left\{f_{a}: a \in A\right\}$ is $\mathfrak{\Im}$-continuous, 1 on $A$ and 0 on $B$.

In conclusion we should like to raise several questions. Does Martin's Axiom imply generalized Lusin subspaces of cometrizable spaces are perfectly normal or countably paracompact? (With regard to countable paracompactness, we note incidentally that our arguments enable us to characterize under Martin's Axiom the countably paracompact subspaces of the density topology. They are in fact the normal subspaces.) Is there a "real" example of a homogeneous normal non-collectionwise Hausdorff space? 
Note added in proof. Zajicek and Lukes have established the cometrizability of Scheinberg's extremally disconnected strengthening of the density topology. It follows that the space $S$ of Theorem 13 may be taken to be extremally disconnected.

\section{REFERENCES}

1. K. Alster and T. Przymusiński, Normality and Martin's Axiom, Fund. Math., 91 (1976), 123-131.

2. R. H. Bing, Metrization of topological spaces, Canad. J. Math., 3 (1951), 175-186.

3. —_ A translation of the normal Moore space conjecture, Proc. Amer. Math. Soc., 16 (1965), 612-619.

4. D. Burke and E. K. van Douwen, No nice dense $G_{8}$ subsets of semimetrizable spaces, in preparation.

5. A. Charlesworth, R. Hodel and F. Tall, On a theorem of Jones and Heath concerning separable normal spaces, Colloq. Math., 34 (1975), 33-37.

6. E. K. van Douwen, Simultaneous extension of continuous functions, Thesis, Free University, Amsterdam, 1975.

$6 . \quad$ - Neighborhood assignments, in preparation.

8. E. K. van Douwen, F. D. Tall and W. A. R. Weiss, Non-metrizable hereditarily Lindelöf spaces with point-countable bases from CH, Proc. Amer. Math. Soc., 64 (1977), 139-145.

9. W. G. Fleissner, When is Jones' space normal?, Proc. Amer. Math. Soc., 50 (1975), 375-378.

10. C. Goffman, C. J. Neugebauer and T. Nishiura, Density topology and approximate continuity, Duke Math. J., 28 (1961), 497-506.

11. R. W. Heath, Screenability, pointwise paracompactness and metrization of Moore spaces, Canad. J. Math., 16 (1964), 763-770.

12. A. Hajnal and I. Juhász, A consistency result concerning hereditarily $\alpha$-Lindelöf spaces, Acta Math. Acad. Sci. Hungar., 24 (1973), 307-312.

13. I. Juhász, Cardinal functions in topology, Mathematical Centre, Amsterdam, 1971.

14. K. Kunen, Luzin spaces, Topology Proc., 1 (1976), 191-199.

15. D. A. Martin and R. M. Solovay, Internal Cohen extensions, Ann. Math. Logic, 2 (1970), 143-178.

16. J. C. Oxtoby, Measure and Category, Springer-Verlag, New York, 1971.

17. T. Przymusiński, $A$ Lindëlof space $X$ such that $X^{2}$ is normal but not paracompact, Fund. Math., 78 (1973), 291-296.

18. T. Przymusiński, Normality and separability of Moore spaces, 325-338 in Set-theoretic Topology; ed. G. M. Reed, Academic Press, New York, 1977.

19. T. Przymusiński and F. D. Tall, The undecidability of the existence of a non-separable normal Moore space satisfying the countable chain condition, Fund. Math., 85 (1974), 291-297.

20. S. Scheinberg, Topologies which generate a complete measure algebra, Advances in Math., 7 (1971), 231-239.

21. F. D. Tall, Set-theoretic consistency results and topological theorems concerning the normal Moore space conjecture and related problems, Thesis, University of Wisconsin, Madison, 1969; Dissert. Math., 144 (1977), 1-53.

22. _ On the existence of non-metrizable metacompact normal Moore spaces, Canad. J. Math., 26 (1974), 1-6.

23. - The countable chain condition versus separability-applications of Martin's Axiom, General Topology and Appl., 4 (1974), 315-339.

24. - The density topology, Pacific J. Math., 62 (1976), 275-284. 
25. - First countable spaces with caliber $\kappa_{1}$ mays or may not be separable, 353-356 in Set-theoretic Topology, ed. G. M. Reed, Academic Press, 1977.

26. H. E. White, Jr., An example involving Baire spaces, Proc. Amer. Math. Soc., 48 (1975), 228-230.

Received November 23, 1976 and in revised form April 22, 1977. The author acknowledges support from Grant A-7354 of the National Research Council of Canada.

UNIVERSITY OF TORONTO

Toronto, Canada 


\section{PACIFIC JOURNAL OF MATHEMATICS}

\section{EDITORS}

RICHARD ARENS (Managing Editor)

University of California

Los Angeles, CA 90024

R. A. BeAumont

University of Washington

Seattle, WA 98105

C. C. MOORE

University of California

Berkeley, CA 94720
J. DUGUNDJI

Department of Mathematics

University of Southern California

Los Angeles, CA 90007

R. FINN AND J. MILGRAM

Stanford University

Stanford, CA 94305

\section{ASSOCIATE EDITORS}
E. F. BECKENBACH
B. H. NEUMANN
F. WOLF
K. YoshidA

\section{SUPPORTING INSTITUTIONS}

UNIVERSITY OF BRITISH COLUMBIA

UNIVERSITY OF SOUTHERN CALIFORNIA

CALIFORNIA INSTITUTE OF TECHNOLOGY

STANFORD UNIVERSITY

UNIVERSITY OF CALIFORNIA

UNIVERSITY OF HAWAII

MONTANA STATE UNIVERSITY

UNIVERSITY OF TOKYO

UNIVERSITY OF NEVADA

UNIVERSITY OF UTAH

NEW MEXICO STATE UNIVERSITY

OREGON STATE UNIVERSITY

UNIVERSITY OF OREGON

OSAKA UNIVERSITY

WASHINGTON STATE UNIVERSITY

UNIVERSITY OF WASHINGTON

AMERICAN MATHEMATICAL SOCIETY

The Supporting Institutions listed above contribute to the cost of publication of this Journal, but they are not owners or publishers and have no responsibility for its contents or policies.

Mathematical papers intended for publication in the Pacific Journal of Mathematics should be in typed form or offset-reproduced (not dittoed), double spaced with large margins. Underline Greek letters in red, German in green, and script in blue. The first paragraph or two must be capable of being used separately as a synopsis of the entire paper. Items of the bibliography should not be cited there unless absolutely necessary, in which case they must be identified by author and Journal, rather than by item number. Manuscripts, in duplicate, may be sent to any one of the four editors. Please classify according to the scheme of Math. Reviews, Index to Vol. 39. All other communications should be addressed to the managing editor, or Elaine Barth, University of California, Los Angeles, California, 90024.

100 reprints are provided free for each article, only if page charges have been substantially paid. Additional copies may be obtained at cost in multiples of 50 .

The Pacific Journal of Mathematics is issued monthly as of January 1966. Regular subscription rate: $\$ 72.00$ a year (6 Vols., 12 issues). Special rate: $\$ 36.00$ a year to individual members of supporting institutions.

Subscriptions, orders for numbers issued in the last three calendar years, and changes of address should be sent to Pacific Journal of Mathematics, 103 Highland Boulevard, Berkeley, California, 94708.

PUBLISHED BY PACIFIC JOURNAL OF MATHEMATICS, A NON-PROFIT CORPORATION

Printed at Jerusalem Academic Press, POB 2390, Jerusalem, Israel. 


\section{Pacific Journal of Mathematics}

\section{Vol. 75, No. $2 \quad$ October, 1978}

Susan Jane Zimmerman Andima and W. J. Thron, Order-induced

topological properties ................................... 297

Gregory Wade Bell, Cohomology of degree 1 and 2 of the Suzuki groups . . 319

Richard Body and Roy Rene Douglas, Rational homotopy and unique

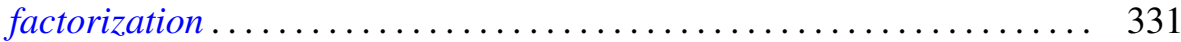

Frank Lewis Capobianco, Fixed sets of involutions ................. 339

L. Carlitz, Some theorems on generalized Dedekind-Rademacher sums .... 347

Mary Rodriguez Embry and Alan Leslie Lambert, The structure of a special class of weighted translation semigroups .....................

Steve Ferry, Strongly regular mappings with compact ANR fibers are

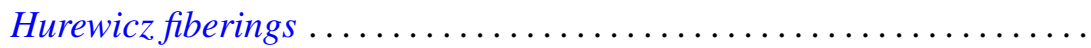

Ivan Filippenko and Marvin David Marcus, On the unitary invariance of the

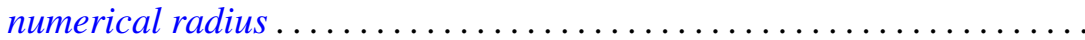

$\mathrm{H}$. Groemer, On the extension of additive functionals on classes of convex

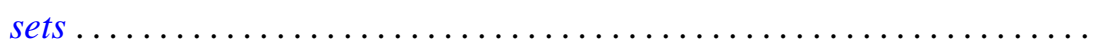

Rita Hall, On the cohomology of Kuga's fiber variety ............... 411

H. B. Hamilton, Congruences on $\mathrm{N}$-semigroups ................. 423

Manfred Herrmann and Rolf Schmidt, Regular sequences and lifting

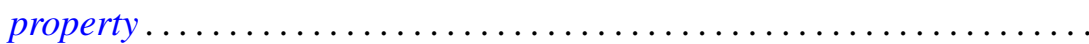

James Edgar Keesling, Decompositions of the Stone-Čech compactification which are shape equivalences .....................

Michael Jay Klass and Lawrence Edward Myers, On stopping rules and the expected supremum of $S_{n} / T_{n}$

Ronald Charles Linton, $\lambda$-large subgroups of $C_{\lambda}$-groups

William Owen Murray, IV and L. Bruce Treybig, Triangulations with the free cell property ............................

Louis Jackson Ratliff, Jr., Polynomial rings and $H_{i}$-local rings ...

Michael Rich, On alternate rings and their attached Jordan rings....

Gary Sampson and H. Tuy, Fourier transforms and their Lipschitz

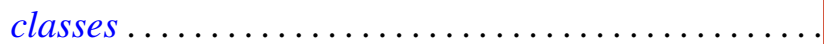

Helga Schirmer, Effluent and noneffluent fixed points on dendrites ...

Daniel Byron Shapiro, Intersections of the space of skew-symmetric maps

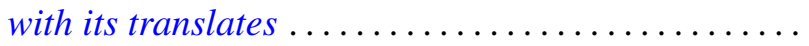

Edwin Spanier, Tautness for Alexander-Spanier cohomology ...

Alan Stein and Ivan Ernest Stux, A mean value theorem for binary digits ...

Franklin D. Tall, Normal subspaces of the density topology . .

William Yslas Vélez, Prime ideal decomposition in $F\left(\mu^{1 / p}\right) \ldots$ 